\title{
Associação entre composição corpo- ral, equilíbrio e mobilidade de idosos com 80 anos ou mais
}

\section{Association between body composition, balance and mobility of elderly aged 80 years or older}

Vanessa R. dos Santos ${ }^{1}$, Diego G. D. Christofaro², Lionai L. dos Santos ${ }^{3}$, Igor C. Gomes ${ }^{4}$, Jamile S. Codogno ${ }^{5}$, Ismael F. Freitas Júnior ${ }^{6}$

\begin{abstract}
RESUMO
Modelo do estudo: Estudo transversal. Objetivo: O objetivo do presente estudo foi analisar a associação entre composição corporal, mobilidade e equilíbrio de idosos com idade igual ou superior a 80 anos. Métodos: A amostra foi composta por 123 idosos com idade entre 80 e 95 anos (83,2 $\pm 2,7$ anos), sendo 78 mulheres $(83,2 \pm 2,9$ anos) e 45 homens $(83,2 \pm 2,4$ anos) residentes na área urbana de Presidente Prudente - SP. A avaliação da composição corporal foi feita pelo método de Absorciometria por Dupla Emissão de Raios-X (DEXA). A mobilidade e o equilíbrio foram avaliados por meio dos testes de velocidade para caminhar, equilíbrio estático e força de membros inferiores. Para tratamento estatístico realizou-se o teste qui-quadrado, o software utilizado foi SPSS (13.0) e o nível de significância estabelecido foi de $5 \%$. Resultados: No grupo masculino, os idosos com maior desempenho nos testes funcionais apresentaram maiores valores percentuais de massa muscular (MM) $(35,6 \%)$ comparados aos de menor desempenho $(15,6 \%), p=0,026$. No grupo feminino, as idosas com maior desempenho nos testes apresentaram maiores valores de densidade mineral óssea (DMO) $(30,8 \%)$ comparadas as de menor desempenho $(20,5 \%) p=0,041$. Conclusão: $A$ MM para idosos do sexo masculino e a DMO para o sexo feminino foram os componentes da composição corporal que se associaram com a capacidade funcional.
\end{abstract}

Palavras-chave: Composição Corporal. Limitação da Mobilidade. Equilíbrio Postural. Idoso de 80 Anos ou mais.

\section{Introdução}

A expectativa de vida vem aumentando na população mundial. O envelhecimento é um processo complexo que altera a morfologia e as funções do or- ganismo e, entre as alterações, estão à mudança na composição corporal ${ }^{1} \mathrm{e}$ a redução da capacidade funcional. $^{2}$

No decorrer do processo, ocorre modificação na composição corporal, com o aumento da massa
1. Mestre em Ciências da Motricidade. Programa de Pós-Graduação em Ciências da Motricidade. (UNESP, Rio Claro/SP. Centro de Estudos e Laboratório de Avaliação e Prescrição de Atividades Motoras(CELAPAM). UNESP, Presidente Prudente/SP.

2. Doutor em Saúde Coletiva - UEL. Departamento de Educação Física. UNESP, Presidente Prudente/SP.

3. Graduanda em Educação Física,UNESP,Presidente Prudente/SP.

4. Mestre em Fisioterapia - UNESP Presidente Prudente/SP

5. Doutora em Ciências da Motricidade - UNESP Rio Claro. Departamento de Educação Física. UNESP, Presidente Prudente/SP.

6. Livre-docente, Departamento de Educação Física; Centro de Estudos e Laboratório de Avaliação e Prescrição de Atividades Motoras(CELAPAM). UNESP, Presidente Prudente/SP.
Correspondência:

Profa. Ms. Vanessa Ribeiro dos Santos Departamento de Educação física. Rua Roberto Simonsen, 305

CEP: 19060-900.Presidente Prudente -SP Telefone: 1832295828 e-mail: van_vrs@yahoo.com.br

Artigo recebido em 16/04/2012 Aprovado para publicação em 12/04/2013 
gorda (MG) e diminuição da MM. A ocorrência dessas duas alterações representa um desafio para os profissionais de saúde, uma vez que necessitam adequar suas ações visando retardar a perda de massa magra e o ganho de massa gorda nos idosos, por constituir um fator de risco para diversas doenças. ${ }^{3}$

Esse aumento da gordura corporal compromete a capacidade funcional ${ }^{4,5}$ e a MM é uma das variáveis mais utilizadas para a indicativo de fragilidade em idosos. A sua diminuição está associada ao maior risco de quedas, aumento das hospitalizações e mortalidade. ${ }^{6}$ Outro componente importante é a densidade mineral óssea (DMO) utilizada para o diagnóstico de osteoporose e na discriminação do risco de fraturas.

As alterações ocorridas na composição corporal estão diretamente relacionadas com a redução da capacidade funcional de indivíduos mais velhos ${ }^{1}$, porém, não está bem estabelecido na literatura qual dos componentes da composição corporal (MM, MG ou DMO) é o mais determinante na capacidade funcional dos idosos. Além disso, há uma carência de estudos que analisem essa relação em idosos com idade igual ou superior a 80 anos. Dessa forma, o objetivo do presente estudo foi analisar a associação entre composição corporal, mobilidade e equilíbrio de idosos com 80 anos ou mais.

\section{Material e Métodos}

\section{Amostragem}

Estudo com delineamento transversal, o qual foi conduzido no período de outubro de 2009 a maio de 2010 em Presidente Prudente (aproximadamente 210.000 habitantes), ${ }^{7}$ localizada a oeste do Estado de São Paulo e com Índice de Desenvolvimento Humano de 0,846 , sendo a $14^{\circ}$ do estado. ${ }^{8}$ De acordo com o Instituto Brasileiro de Geografia e Estatística ${ }^{9}$ a projeção para o ano de 2009 de brasileiros com idade igual ou acima de 80 anos era de 2.653.060, representando $1,1 \%$ da população, partindo desse princípio a estimativa de idosos nessa faixa etária na cidade de Presidente Prudente era de cerca de 2.100 habitantes. Para composição da amostra adotou-se uma margem de erro de $3 \%$ e uma aceitação de $60 \%$ de participação na pesquisa.

A secretaria municipal de saúde da cidade disponibilizou nome, endereço e telefone dos indivíduos com 80 anos ou mais. Com essas informações foi realizado sorteio de maneira aleatória de 226 indivíduos e a abordagem foi realizada por meio de telefone. Fo- ram excluídos da amostra os indivíduos que não deambulavam, acamados, residentes da área rural, institucionalizados, portadores de marca passo e os indivíduos com dados incompletos. Sendo assim, a amostra para o presente estudo foi composta por 123 indivíduos de ambos os sexos, e desses participantes $65 \%$ eram hipertensos, $19 \%$ apresentavam dislipidemia e $19 \%$ diabetes mellitus.

Todas as avaliações foram realizadas por monitores treinados e capacitados do Centro de estudos e Laboratório de Avaliação e Prescrição de Atividade Motora (CELAPAM), do Departamento de Educação Física da Universidade Estadual Paulista, Campus de Presidente Prudente.

Os indivíduos convidados a participar do presente estudo foram esclarecidos sobre os objetivos e a metodologia empregada para a coleta dos dados. Somente os que assinaram o "Termo de Consentimento Livre e Esclarecido" fizeram parte da amostra. O projeto foi aprovado pelo Comitê de Ética em pesquisa da Universidade Estadual Paulista (Processo n 26/2009).

\section{Antropometria e Composição Corporal}

Para avaliação da massa corporal foi utilizada uma balança digital, marca Filizola, precisão de $0,1 \mathrm{~kg}$ e capacidade máxima de $150 \mathrm{~kg}$. Os idosos usaram roupas leves, permaneceram descalços e foram posicionados em pé, no centro da plataforma da balança. A estatura foi medida com estadiômetro de metal, fixo, precisão de $0,1 \mathrm{~cm}$ e extensão máxima de dois metros. Todas as medidas antropométricas foram realizadas seguindo a padronização descrita por Freitas Jr. et. al. ${ }^{10}$

Para análise da composição corporal foi utilizado o aparelho de Absortiometria de Raios-X de Dupla Energia (DEXA) da marca Lunar, modelo DPX-MD, software 4,7 que utiliza o modelo de três compartimentos (massa corporal magra (MM), massa de gordura (MG) e densidade mineral corporal (DMO). Esta técnica permite estimar a composição corporal total e por segmento. $\mathrm{O}$ avaliado deve permanecer posicionado em decúbito dorsal, sem se movimentar no aparelho durante a realização da medida. Os resultados foram transmitidos ao computador interligado ao aparelho, o qual forneceu informações sobre: MM $(\mathrm{kg})$, $\mathrm{MG}(\mathrm{kg})$ e DMO $\left(\mathrm{g} / \mathrm{cm}^{2}\right)$.

\section{Equilíbrio e mobilidade}

O equilíbrio e a mobilidade dos idosos foram analisados por meio dos testes de equilíbrio estático, velocidade para caminhar e força de membros infe- 
riores presentes na seção $L$ do questionário do projeto Saúde, bem estar e envelhecimento (SABE).$^{11} \mathrm{O}$ teste de equilíbrio possui três etapas realizadas em sequência (10 segundos cada): i) ficar em pé, com os pés unidos; ii) encostar o calcanhar de um dos pés na lateral do hálux do pé oposto, iii) ficar em pé, com pé na frente do outro. A pontuação das três etapas foram somadas e a partir da soma foi obtido o resultado final.

No teste de caminhada o idoso caminhou por 3 metros na mesma velocidade utilizada para realizar as suas atividades cotidianas e o tempo do percurso foi cronometrado e registrado pelos avaliadores. Para avaliação da força de membros inferiores foi aplicado o teste de levantar/sentar da cadeira onde o idoso manteve os braços cruzados sobre o peito e, ao sinal do avaliador, levantou e sentou na cadeira, o mais rápido possível, cinco vezes em menos de 60 segundos.

A pontuação de cada teste varia entre 0 e 4 pontos. Para a classificação do desempenho dos idosos foi utilizada a soma dos pontos obtidos nos três testes.

\section{Análise Estatística}

Para as variáveis numéricas, a normalidade do conjunto de dados analisado foi confirmada pelo teste de Komolgorov-Smirnov (K-S). Assim, a estatística descritiva foi composta por valores de média (tendência central) e desvio-padrão (dispersão). Os valores médios de cada variável foram comparados, segundo sexo, por meio do teste $t$ de Student para amostras independentes.
As variáveis da composição corporal, equilíbrio e mobilidade foram categorizadas a partir dos valores de mediana, sendo considerados, idosos com maior MG, MM e DMO aqueles que estavam acima dos valores de mediana e com menores valores aqueles abaixo da mediana. A pontuação total obtida nos três testes (0-12 pontos), também foi classificada segundo valor de mediana, sendo considerados idosos com menor equilíbrio e mobilidade aqueles que obtiveram (0-5 pontos) e maior (6-12 pontos). O teste qui-quadrado foi utilizado para testar a associação entre o desempenho nos testes e a composição corporal. $\mathrm{O}$ tratamento estatístico foi realizado utilizando o software SPSS (SPSS inc. Chicago. IL), versão 13.0 e o nível de significância foi estabelecido em $5 \%$.

\section{Resultados}

A Tabela 1 apresenta as características gerais e variáveis da composição corporal da amostra estudada, estratificada por sexo. Não houve diferença na idade dos idosos participantes do estudo. Os homens apresentaram valores de peso, estatura, DMO, MM em quilos e proporcional ao peso corporal significativamente superiores. A MG em quilos não apresentou diferença estatística entre os sexos, no entanto, em valores proporcionais foi maior para as mulheres.

Na Tabela 2 são apresentados os valores percentuais da associação entre as variáveis da composição corporal, mobilidade e equilíbrio. Os idosos com

\section{Tabela 1}

Variáveis descritivas da amostra, segundo o sexo.

\begin{tabular}{|c|c|c|c|c|c|c|c|c|}
\hline \multirow[b]{2}{*}{ Variáveis } & \multicolumn{3}{|c|}{$\begin{array}{c}\text { Masculino } \\
(n=45)\end{array}$} & \multicolumn{3}{|c|}{$\begin{array}{c}\text { Feminino } \\
(n=78)\end{array}$} & \multirow[b]{2}{*}{$\mathbf{t}$} & \multirow[b]{2}{*}{$\mathbf{P}^{*}$} \\
\hline & Média & \pm & DP & Média & \pm & DP & & \\
\hline Idade (anos) & 83,2 & \pm & 2,4 & 83,2 & \pm & 2,9 & $-0,071$ & 0,943 \\
\hline Peso $(\mathrm{Kg})$ & 72,8 & \pm & 16,9 & 58,9 & \pm & 10,9 & 5,541 & 0,001 \\
\hline Estatura $(\mathrm{cm})$ & 165,0 & \pm & 8,0 & 150,8 & \pm & 7,1 & 10,192 & 0,001 \\
\hline Massa Magra (Kg) & 46,770 & \pm & 10,9 & 31,453 & \pm & 6,0 & 10,039 & 0,001 \\
\hline Massa Magra (\%) & 66,6 & \pm & 8,7 & 55,8 & \pm & 8,3 & 6,860 & 0,000 \\
\hline Massa Gorda (Kg) & 20,701 & \pm & 9,3 & 23,389 & \pm & 8,7 & $-1,608$ & 0,110 \\
\hline Massa Gorda (\%) & 29,5 & \pm & 8,9 & 40,3 & \pm & 8,5 & $-6,696$ & 0,000 \\
\hline $\operatorname{DMO}\left(\mathrm{g} / \mathrm{cm}^{2}\right)$ & 1,13 & \pm & 0,13 & 0,97 & \pm & 0,11 & 7,371 & 0,001 \\
\hline
\end{tabular}

${ }^{*}=$ teste t de Student para dados independentes; $\mathrm{DP}=$ desvio-padrão; $\mathrm{DMO}=$ densidade mineral óssea. 
maiores valores de $\mathrm{MM}(\mathrm{Kg})$ apresentaram maior desempenho nos testes funcionais (Alta $\mathrm{MM}(\mathrm{Kg}): 60,3 \%$ versus Baixa $\mathrm{MM}(\mathrm{Kg}): 40 \%$; $\mathrm{p}=0,024)$. Padrão similar foi observado para maiores valores de DMO (Alta DMO: 61,9\% versus Baixa DMO: 38,3\%; $\mathrm{p}=0,009)$.

No grupo masculino, os idosos com maior desempenho nos testes funcionais apresentaram maio- res valores de $\mathrm{MM}(\mathrm{Kg})$ comparados aos de menor desempenho ( $\mathrm{p}=0,026)$. E no grupo feminino, a variável da composição corporal que apresentou associação com o desempenho nos testes foi a DMO, sendo as idosas com maior desempenho apresentaram maiores valores de DMO comparadas as de menor desempenho $(\mathrm{p}=0,041)$ (Tabela 3$)$.

\section{Tabela 2}

Associação entre os componentes da composição corporal, mobilidade e equilíbrio de idosos maiores de 80 anos.

\begin{tabular}{|c|c|c|c|c|}
\hline \multirow[b]{2}{*}{ Composição Corporal } & & \multicolumn{2}{|c|}{ Desempenho nos testes } & \multirow[b]{2}{*}{$P$} \\
\hline & & Menor & Maior & \\
\hline \multirow[t]{2}{*}{$\mathrm{MM}(\mathrm{Kg})$} & Baixa & $60,0 \%$ & $40,0 \%$ & $0,024^{*}$ \\
\hline & Alta & $39,7 \%$ & $60,3 \%$ & \\
\hline \multirow[t]{2}{*}{$\mathrm{MM}(\%)$} & Baixa & $49,2 \%$ & $50,8 \%$ & 0,928 \\
\hline & Alta & $50,0 \%$ & $50,0 \%$ & \\
\hline \multirow[t]{2}{*}{$\mathrm{MG}(\mathrm{Kg})$} & Baixa & $54,1 \%$ & $45,9 \%$ & 0,322 \\
\hline & Alta & $45,2 \%$ & $54,8 \%$ & \\
\hline \multirow[t]{2}{*}{$\operatorname{MG}(\%)$} & Baixa & $51,6 \%$ & $48,4 \%$ & 0,652 \\
\hline & Alta & $47,5 \%$ & $52,5 \%$ & \\
\hline \multirow[t]{2}{*}{$\mathrm{DMO}(\mathrm{g} / \mathrm{cm} 2)$} & Baixa & $61,7 \%$ & $38,3 \%$ & $0,009 *$ \\
\hline & Alta & $38,1 \%$ & $61,9 \%$ & \\
\hline
\end{tabular}

*= teste qui-quadrado com valor crítico significativo; $\mathrm{MM}=$ massa magra; $\mathrm{MG}=$ massa gorda; $\mathrm{DMO}=$ densidade mineral óssea.

Tabela 3

Associação entre a composição corporal, mobilidade e equilíbrio, segundo sexo.

\begin{tabular}{|c|c|c|c|c|c|c|c|}
\hline \multirow[b]{2}{*}{ Composição Corporal } & & \multicolumn{2}{|c|}{$\begin{array}{c}\text { Masculino } \\
\text { Desempenho nos testes }(\%)\end{array}$} & \multirow[b]{2}{*}{$P$} & \multicolumn{2}{|c|}{$\begin{array}{c}\text { Feminino } \\
\text { Desempenho nos testes }(\%)\end{array}$} & \multirow[b]{2}{*}{$P$} \\
\hline & & Menor & Maior & & Menor & Maior & \\
\hline \multirow[t]{2}{*}{$\mathrm{MM}(\mathrm{Kg})$} & Baixo & 63,6 & 36,4 & 0,026 & 57,9 & 42,1 & 0,255 \\
\hline & Alto & 30,4 & 69,6 & & 45,0 & 55,0 & \\
\hline \multirow[t]{2}{*}{$\operatorname{MM}(\%)$} & Baixo & 50,0 & 50,0 & 0,661 & 48,7 & 51,3 & 0,651 \\
\hline & Alto & 43,5 & 56,5 & & 53,8 & 46,2 & \\
\hline \multirow[t]{2}{*}{$\mathrm{MG}(\mathrm{Kg})$} & Baixo & 50,0 & 50,0 & 0,661 & 56,4 & 43,6 & 0,365 \\
\hline & Alto & 43,5 & 56,5 & & 46,2 & 53,8 & \\
\hline \multirow[t]{2}{*}{$\mathrm{MG}(\%)$} & Baixo & 47,8 & 52,2 & 0,873 & 53,8 & 46,2 & 0,651 \\
\hline & Alto & 45,5 & 54,5 & & 48,7 & 51,3 & \\
\hline \multirow[t]{2}{*}{$\operatorname{DMO}\left(\mathrm{g} / \mathrm{cm}^{2}\right)$} & Baixo & 59,1 & 40,9 & 0,102 & 63,2 & 36,8 & $0,041^{*}$ \\
\hline & Alto & 34,8 & 65,2 & & 40,0 & 60,0 & \\
\hline
\end{tabular}

*= teste qui-quadrado com valor crítico significativo; $\mathrm{MM}=$ massa magra; $\mathrm{MG}=$ massa gorda; $\mathrm{DMO}=$ densidade mineral óssea. 


\section{Discussão}

A redução progressiva da $\mathrm{MM}$ em idosos é uma preocupação constante para os profissionais da saúde, devido sua relação com a incapacidade física, mobilidade e mortalidade. ${ }^{12,13,14}$ Em nosso estudo, apesar de não ter sido observada associação entre a MM proporcional ao peso corporal total, equilíbrio e mobilidade, foi observado que os idosos com maiores valores de $\mathrm{MM}(\mathrm{Kg})$ obtiveram melhor desempenho nos testes para mobilidade e equilíbrio comparados aos com menores valores de $\mathrm{MM}(\mathrm{Kg})$. Quando analisada a amostra estratificada segundo sexo, esta associação foi observada apenas no sexo masculino. Aparentemente, esta associação é dependente do sexo, uma vez que ambos diferem muito ao longo da vida no que se refere ao ganho e perda de MM. Assim, estratégias de aumento da MM devem estar atentas às diferenças inerentes ao sexo.

Além, de aumentar o risco de doença coronariana, também foi evidenciado que o excesso de gordura corporal em idosos limita a mobilidade. ${ }^{15}$ Isso acontece em virtude da maior quantidade de massa gorda ou a maior proporção de gordura corporal, que podem aumentar a sobrecarga corporal, limitando os movimentos e aumentando o estresse nas articulações e músculos, acentuando o risco de incapacidade nos idosos com excesso de gordura. ${ }^{16}$

Em nosso estudo não foi encontrada associação entre o desempenho nos testes de equilíbrio e mobilidade e a quantidade de MG em idosos longevos. Similarmente, no estudo de Sallinen et al. ${ }^{17}$ a associação entre o percentual de gordura corporal e a velocidade máxima de caminhada foi mais significativa para indivíduos com idade entre 60 e 79 anos, enquanto que para idosos maiores de 80 anos essa associação não foi muito evidente. Esses resultados podem dar um indicativo de que, a quantidade de gordura corporal pode não exercer tanta influência sobre a mobilidade de idosos mais velhos, uma vez que, a massa gorda também tende a diminuir com o envelhecimento, e essa diminuição parece ser devido, principalmente, à perda de gordura subcutânea. ${ }^{1}$

Recentemente, vários autores têm investigado o efeito da combinação de ambas as condições (redução da massa e força muscular e aumento da gordura corporal) em indivíduos mais velhos. Essa condição, definida como obesidade sarcopênica, está sendo considerada mais prejudicial à mobilidade, equilíbrio e a saúde geral do idoso. No estudo longitudinal conduzido por Stenholm et al., ${ }^{18}$ foi observado que a presença de obesidade sarcopênica, aumenta o risco de declínio na velocidade de caminhada e deficiência na mobilidade de idosos maiores de 65 anos. Bouchard e Janssen ${ }^{19}$ observaram que indivíduos acima de 55 anos, com menor força muscular e obesidade apresentaram função física inferior do que aqueles com apenas obesidade. No presente estudo, apenas a variável massa muscular magra em idosos do sexo masculino apresentou associação positiva com a mobilidade e equilíbrio, e a gordura corporal não apresentou significância em ambos os sexos. Esses resultados indicam que, aparentemente, a perda de MM é mais prejudicial à mobilidade e equilíbrio do que o excesso de MG em idosos longevos do sexo masculino.

A massa óssea diminui com o envelhecimento, principalmente, em mulheres ${ }^{1}$ após a menopausa. A redução da massa muscular e óssea exerce influência sobre os estados nutricional, endócrino, funcional e cognitivo, bem como propícia comorbidades. ${ }^{1} \mathrm{Com}$ o comprometimento ósseo em regiões importantes como, por exemplo, o fêmur e a coluna lombar, que auxiliam na locomoção, postura e equilíbrio, a execução de algumas atividades motoras torna-se dificultadas. Nossos achados estão de acordo com a literatura e indicam que mulheres maiores de 80 anos, com menor densidade óssea, também possuem menor mobilidade e equilíbrio. Esse é um achado preocupante, uma vez que a diminuição da mobilidade e do equilíbrio está associada a um maior número de quedas em idosos do sexo feminino. ${ }^{20}$

O delineamento transversal do estudo limita a capacidade de estabelecer relações de causalidade e, dessa forma, os achados precisam ser analisado sob esta condição. Além disso, indica-se a necessidade de se conduzir experimentos prospectivos envolvendo a temática.

Em conclusão, a MM para idosos do sexo masculino e a DMO para o sexo feminino foram os componentes da composição corporal que se associaram com a mobilidade e equilíbrio. Exercícios físicos que almejem a preservação da massa muscular e óssea devem ser incentivados para idosos longevos. 


\section{ABSTRACT}

Study design: cross-sectional study. Objective: The objective of this study was to analyze the association between body composition, mobility and balance of elderly aged 80 years or older. Methods: The sample consisted of 123 elderly aged 80 and 95 years $(83.2 \pm 2.7$ years) with 78 women (83.2 \pm 2.9 years) and 45 men (83.2 \pm 2 , 4 years) residing in the city of Presidente Prudente - SP. Assessment of body composition was made by absorpiometria dual energy X-ray (DXA). The mobility and balance were assessed by means of tests of speed walking, and static balance lower limb strength. For statistical analysis we carried out the chi-square test, the software used was SPSS (13.0) and the significance level was set at $5 \%$. Results: In males, those with higher performance in the functional tests showed higher percentages of muscle mass (MM) (35.6\%) compared to lower performance (15.6\%), $\mathrm{p}=0.026$. In the female group, the elderly with higher performance on tests showed higher bone mineral density (BMD) values $(30.8 \%)$ compared with those with lower performance $(20.5 \%) p=0.041$. Conclusion: MM for elderly males and BMD for females were the components of body composition that is associated with functional capacity.

Keywords: Body Composition. Mobility Limitation. Postural Balance. Aged, 80 and over.

\section{Referências}

1. Buffa R, Floris GU, Putzu PF, Marini E. Body Composition Variations in Ageing. Coll Antropol. 2011; 35: 259-65.

2. Murabito JM, Pencina MJ, Zhu L, Kelly-Hayes M, Shrader P, D'agostino RB. Temporal trends in self-reported functional limitations and physical disability among the community-dwelling elderly population: the Framingham hearth study. Am J Public Health. 2008; 98:1256-62.

3. Weinheimer EM, Sands LP, Campbell WW. A systematic review of the separate and combined effects of energy restriction and exercise on fat-free mass in middle-aged and older adults: implications for sarcopenic obesity. Nutr Rev. 2010; 68:375-88.

4. Rech CR, Cruz JLS, Araújo EDS, Kalinowski FG, Dellagrana RA. Associação entre aptidão funcional e excesso de peso em mulheres idosas. Motricidade. 2010; 6:47-53.

5. Arnold AM, Newman AB, Cushman A, Ding J, Kritchevsky S. Body weight dynamics and their association with physical function, and mortality in older adults: the Cardiovascular Health Study. J Gerontol A Biol Sci Med Sci. 2010; 65:63-70.

6. Silva TAA, Frisoli Junior A, Pinheiro MM, Szejnfeld VL. Sarcopenia associada ao envelhecimento: aspectos etiológicos e opções terapêuticas. Rev Bras Reumatol. 2006; 46:391-7.

7. IBGE - Instituto Brasileiro de Geografia e Estatística. Censo Demográfico e Contagem da População: População residente por sexo, situação e grupos de idade, 2010. Disponível em: $<w w w . s i d r a . i b g e . g o v . b r>$ [2011 Dez 5].

8. IBGE - Instituto Brasileiro de Geografia e Estatística. Censo Demográfico e Contagem da População: População residente por sexo, situação e grupos de idade, 2000. Disponível em: $<$ www.sidra.ibge.gov.br> [2011 Dez 5].

9. IBGE - Instituto Brasileiro de Geografia e Estatística. Projeção da população do Brasil por sexo e idade para o período 1980-2050: revisão 2004. Rio de Janeiro: IBGE - Depis. Disponível em: <http://www.ibge.gov.br> [2011 Dez 5].
10. Freitas Junior IF, Bueno DR, Buonani C, Codogno JS, Conterato I, Fernandes RA et. al. Padronização de Técnicas Antropométricas. Presidente Prudente, SP: Cultura Acadêmica; 2009.

11. Lebrão ML, Laurenti R. Saúde, bem-estar e envelhecimento: o estudo SABE no município de São Paulo. Rev Bras Epidemiol. 2005; 8:127-41.

12. Meng-Yueh C, Hsu-Ko K.,Ying-Tai W. Sarcopenia, Cardiopulmonary Fitness, and Physical Disability in Community-Dwelling Elderly People. Phys Ther. 2010; 90:1277-87.

13. Reid KF, Naumova EN, Carabello RJ, Phillips EM, Fielding RA. Lower extremity muscle mass predicts functional performance in mobility-limited elders. J Nutr Health Aging. 2008; 20:493-8.

14. Bunout D, De La Maza MP, Barrera G, Leiva L, Hirsch S. Association between sarcopenia and mortality in healthy older people. Australas J Ageing. 2011; 30:89-92.

15. Stenholm S, Rantanen T, Alanen E, Reunanen A, Sainio P, Koskinen S. Obesity history as a predictor of walking limitation at old age. Obesity (Silver Spring). 2007; 15: 929-38.

16. Visser M, Deeg DJH, Lips P, Harris TB, Bouter LM. Skeletal muscle mass and muscle strength in relation to lower-extremity performance in older men and women. J Am Geriatr. Soc 2000;48:381-6.

17. Sallinen J., Stenholm S., Rantanen T., Helioaara M., Sainio P., Koskinen S. Effect of age on the association between body fat percentage and maximal walking speed. J Nutr Health Aging. 2011; 15:427-32.

18. Stenholm S, Alley D, Bandinelli S, Griswold ME, Koskinen S, Rantanen $T$ et. al. The effect of obesity combined with low muscle strength on decline in mobility in older persons: results from the InChianti Study. Int J Obes. 2009; 33:635-44.

19. Bouchard DR, Janssen I. Dynapenic-obesity and physical function in older adults. J Gerontol A Biol Sci Med Sci. 2010; 65: 71-7.

20. Fabrício SCC, Rodrigues RAP, Costa Jr. ML. Causas e conseqüências de quedas de idosos atendidos em hospital público. Rev Saúde Pública. 2004; 38:93-9. 